\title{
An animal model of acute otitis media and the histopathological assessment of a cochlear implant in the cat
}

by

\author{
Warwick J. Brennan and Graeme M. Clark (Melbourne, Australia)
}

\section{Introduction}

A reliable experimental model of acute otitis media is required to study the spread of middle ear infection to the inner ear in the case of implanted cochleae. Surprisingly for a disease with such a high incidence there have been relatively few experimental studies of otitis media. Some research workers have employed techniques for plugging the Eustachian tube to investigate secretory otitis media. Giebink et al. (1980) have induced pneumococcal otitis media in chinchillas by direct inoculation of bacterial suspensions into the middle ear, and by nasal inoculation followed by a negative pressure applied to the middle ear through an opening in the bulla. Paparella et al. (1980) have examined the round window membrane as it is a likely pathway for the spread of infection from the middle to inner ear. Kawabata and Paparella (1971) have shown details of the three layers of this membrane, and Paparella et al. (1980) have demonstrated the semi-permeable nature of the membrane, and its permeability to tritiated albumin and staphylococcal exotoxin.

The resistance of the implanted round window membrane to the spread of staphylococcal and streptococcal infections in the middle ear has been studied by inoculating bacteria (Shepherd et al. 1984; Clark and Shepherd, 1984) directly into the bulla. The permeability of the implanted round window membrane following middle ear infection has also been examined using horseradish peroxidase (Franz et al. 1984). As an otitis media was not demonstrated in all the animals inoculated in these studies it was considered important to find a more reliable method of producing an infection.

The aim of the present study has been to develop a means of inducing a marked acute otitis media in the experimental animal (in this case cats) which would help determine whether the cochlear implant round window membrane seal can prevent infection in the middle ear spreading to the inner ear structures. A technique has been devised which simulates the pathogenesis of otitis media. It involves a combined trans-Eustachian tube inoculation of bacteria, and tubal occlusion with teflon paste via a transpalatal approach.

The study has demonstrated the marked protective function of the round window membrane against both middle ear infection and the placement of an electrode. It further attests to the ability of the intracochlear implant tissue reaction to withstand the threat of a subsequent middle ear infection.

\section{Method}

Four healthy adult cats of $2.5-4 \mathrm{~kg}$. weight were used. Tympanic membranes were care- 
fully assessed both pre- and post-operatively. Procedures were performed under general anaesthesia using Ketamine hydrochloride (20 mg./kg. ip) and Rompun* $(2.5 \mathrm{mg} . / \mathrm{kg}$. ip). All animal procedures conformed with 'The Code of Practice for the Care and Use of Animals in Research in Australia' set down by the National Health \& Medical Research Council.

Surgery was performed under sterile conditions. The palatal region was infiltrated with $0.2 \mathrm{ml}$. of xylocaine 2 per cent and adrenaline $1: 80,000$ to minimize bleeding. A horizontal transpalatal incision was made just posterior to the junction between the soft and hard palates. The incision gave a good exposure of both Eustachian tubes. The Eustachian tubes were cannulated with a light weight tube of hexafluoropropylene (O.D. $1.1 \mathrm{~mm}$.) under magnification. Extra force was required to introduce the cannula through the isthmus. $0.25 \mathrm{ml}$. of a human staphylococcus aureus broth $\left(10^{4}\right.$ organisms $\left./ \mathrm{ml}\right)$ was inoculated into the middle ear cavity via the tube, using a tuberculin syringe. The tube was then withdrawn and the Eustachian tube orifice sealed by infiltrating $0.3 \mathrm{ml}$. of Polytef pastet into the surrounding mucosa. Adequate closure was confirmed by inability to recannulate the Eustachian tube. The palatal incision was closed with 3.0 chromic catgut. Throughout the procedure care was taken to ensure an adequate and blood-free airway.

Electrodes for implantation were made similar to the specifications of the University of Melbourne's Department of Otolaryngology (Clark et al. 1979). They consisted of a Silastic ${ }^{\circledR}$ shaft and tip with a platinum wire core and two platinum electrode bands. The electrodes were inserted into the round window via the bulla. Under magnification a fine perforation was made in the round window membrane using a 25 gauge needle. The electrode was then gently introduced and fed into the cochle $\mathrm{z}$ up to the beginning of the second platinum band. The electrode was secured in place in an ' $L$ '-shaped recess in the bulla margin, and held with a small muscle plug. The incision was closed using 3.0 chromic catgut in two layers. Implanted animals were given Ampicillin intra-operatively $(500 \mathrm{mg}$. imi) and Amoxicillin for 2-3 days post-operatively (250 mg. orally daily.)

After two weeks the animals were sacrificed under a general anaesthetic using Ketamine hydrochloride, Rompun (ip) and pentobarbitone sodium $(60 \mathrm{mg} . / \mathrm{ml}$., imi). The animals were initially perfused with $0.1 \mathrm{~m}$. phosphate buffered saline containing heparin and then Karnovsky's glutaraldehyde-formaldehyde fixative in $0.1 \mathrm{~m}$. phosphate buffer. Temporal bones were dissected out and placed in neutral buffered formaldehyde, and fixed overnight at $4^{\circ} \mathrm{C}$. The otic capsules were thinned using a diamond burr, and decalcified in EDTA for 5 days. They were washed in distilled water and dehydrated in acetone baths. They were then placed in increasing concentrations of Spurr's epoxy resin in acetone. Embedding took place by polymerizing the Spurr's resin by heating at $70^{\circ} \mathrm{C}$ for 12 hours. Sections of $2 \mu$ thickness were cut at $130 \mu$ intervals. Spurr's resin was dissolved by immersion in saturated sodium hydroxide in ethyl alcohol for 15 minutes. Sections were stained with haematoxylin and eosin.

\section{Results}

A series of three experiments wert performed.

\section{Experiment 1:}

This was a study of the transpalatal middle ear inoculation technique using teflon paste to occlude the Eustachian tube. It was undertaken on the left side in two cats. The tympanic membranes were normal pre-operatively, but immediately after the procedure a discharge was detected in the external ear canal in each cat. The animals were sacrificed two weeks later. The left bulla mucosae appeared thickened. Histological section displayed a marked acute inflammation in the bulla mucosae. The round windows were, however, intact and the cochleas free of any involvement (Fig. 1).

\footnotetext{
* Rompun-Bayer Australia Ltd. 46-47 Wilson St., Botany, N.S.W.

† Ethicon-1/5 Khartoum Road, North Ryde, N.S.W. 2113.
} 


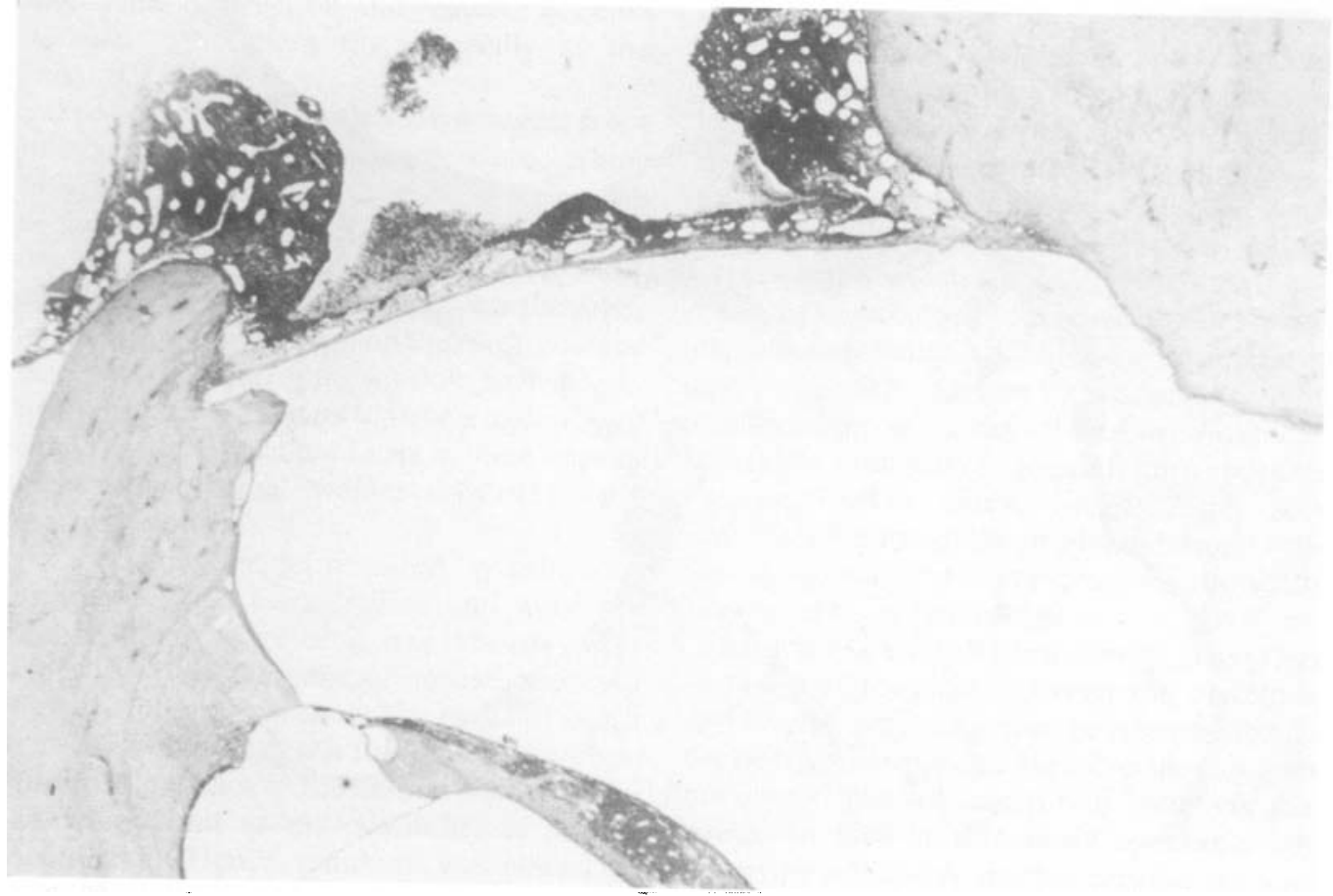

FIG. 1

A photomicrograph of an unimplanted cat cochlea 14 days after the inoculation of a staphylococcus aureus suspension. Magnification $\times 50$.

\section{Experiment 2:}

This was designed to examine the round window and intracochlear reaction to the presence of a cochlear implant electrode in the absence of infection. In one cat both bullae were opened and the cochleae implanted with electrodes. Sacrifice took place seven weeks later. Histology showed an undisturbed round window membrane perforated by a cochlear implant electrode. There was a mesothelial lining next to this electrode, which was surrounded by a fibrous tissue sheath (Fig. 2).

\section{Experiment 3:}

The left bulla of an animal was opened and an electrode implanted into the cochlea. Six weeks later both middle ears were inoculated and the Eustachian tubes occluded. Two weeks later the animal was sacrificed. Histologically the implanted ear showed an acute inflammatory response in the bulla mucosa, but there was no sign of any intracochlear extension (Fig. 3). In the control ear the bulla was inflamed and the cochlea was uninvolved.

\section{Discussion}

This particular model was developed to be consistent with the pathogenesis of otitis media, and to study problems associated with otitis media in implanted cochleae postoperatively.

Similarities with the pathogenesis of otitis media were: the route of introduction of organisms via the nasopharynx, an element of Eustachian tube damage, and Eustachian tube obstruction. It was assumed that the cannulation of the Eustachian tube produced 


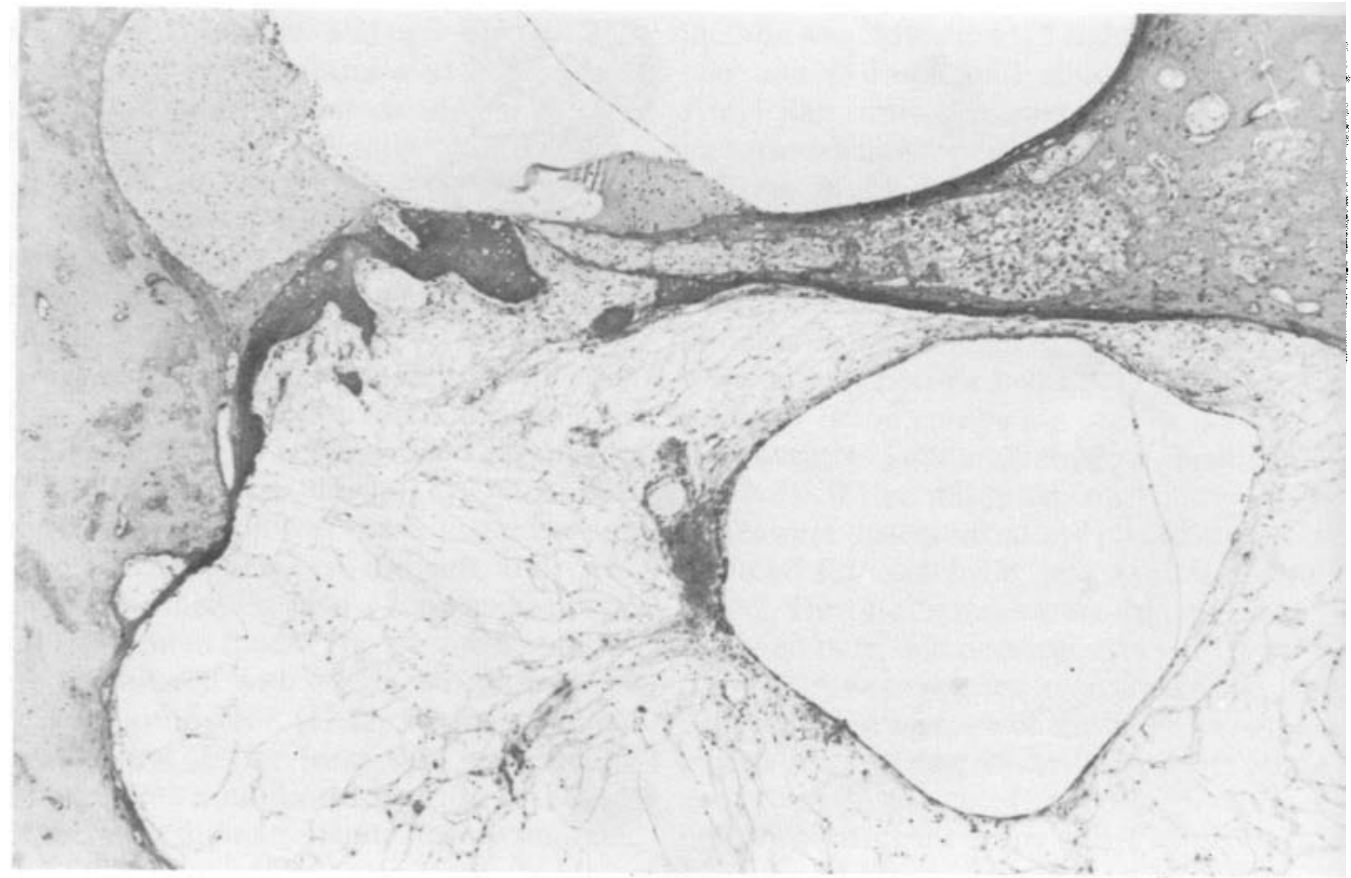

Fig. 2

A photomicrograph of an implanted cat cochlea without bacterial inoculation. Magnification $\times 80$.

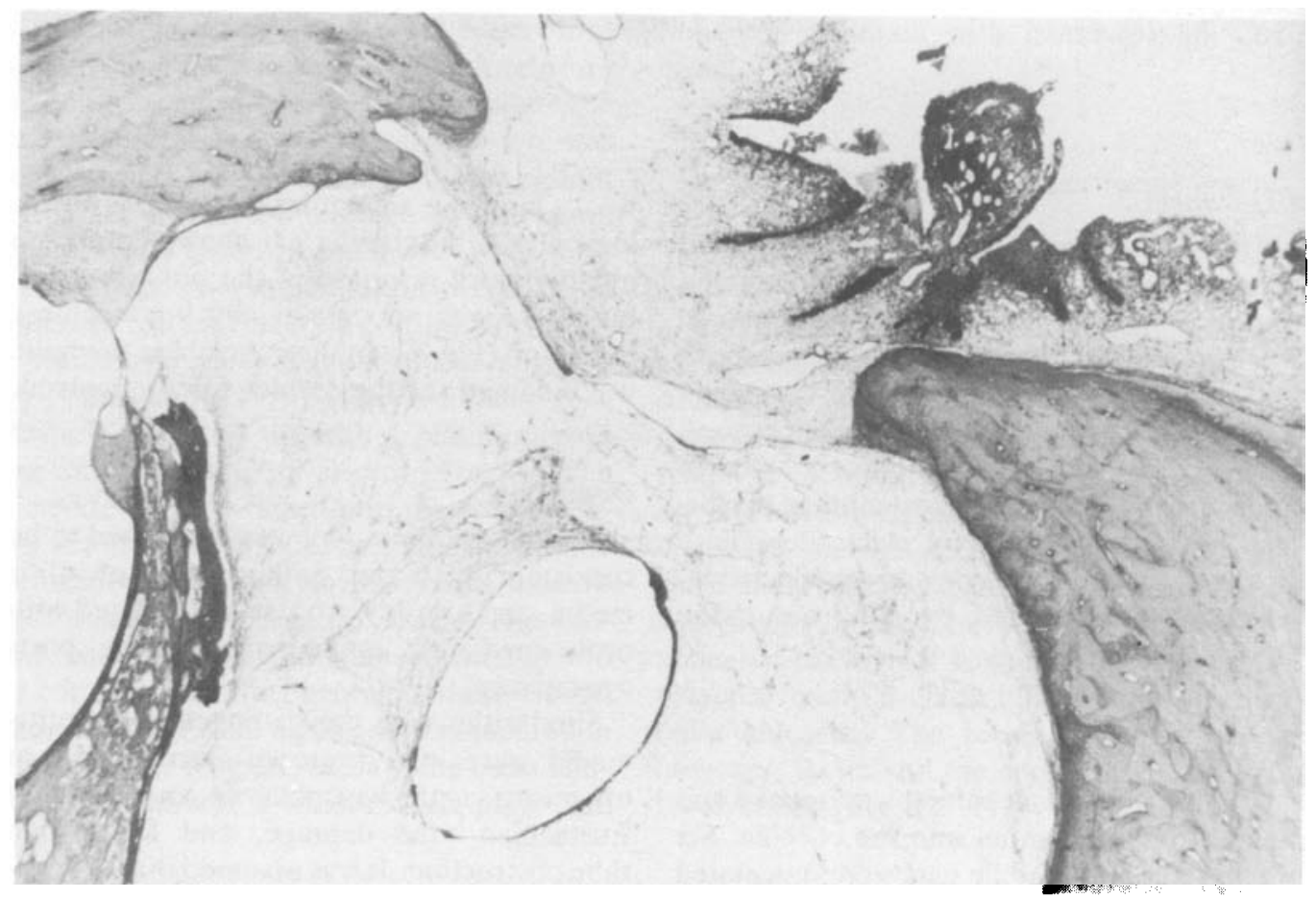

Fig. 3

A photomicrograph of an implanted cat cochlea 14 days after the inoculation of a staphylococcus aureus suspension. Magnification $\times 50$. 
some cilial destruction and reactive oedema because of its tight fit, especially at the isthmus.

The model also has other advantages. It is a relatively straightforward procedure, taking only about five minutes to perform on the anaesthetized animal. It also avoids any risk of disturbing the previously implanted electrode. Inoculation via the bulla, on the other hand, may cause traction on the electrode and disruption of the round window seal. Moreover, the model appears to have a high degree of success (all inoculated ears in these experiments developing well-established acute inflammations).

Care, however, is required in using the technique. Two animals died and were not included in the results; one shortly after recovery from the general anaesthetic, and the other three days later. The cause of death was uncertain, but both timing and the presence of blood clot in the animal airway in the first case point to the possibility of airway obstruction. Hence, emphasis was placed on clearing the airway thoroughly, both during and after the procedure. The timing of the death of the second case, the use of a large number of organisms $\left(10^{6}\right.$ organisms $/ \mathrm{ml}$.), and the animal's general condition suggest the possibility of septicaemia.

Using a partly dissected temporal bone it was shown that if the cannulating tube was forced along the Eustachian tube to its full extent the tip of the tube displaced the malleus laterally, and resulted in a tiny slit-like perforation in the drum. This was the cause of the post-operative canal discharge. The amount of drainage was, however, minimal, and the perforation sealed quickly and did not seem to impair the establishment of an inflammatory response.

Microbiological studies of the middle ear exudates and phage typing of the inoculated staphylococcus were not undertaken as our aim was simply to induce a marked middle ear inflammation with the organism. However, these aspects will be studied in the future as well as the suitability of various animal and human pathogens in inducing animal otitis media.

In the presence of a cochlear implant elec- trode a protective mesothelial reaction in the scala tympani was seen to completely line the surface of the electrode. Surrounding it was a loose fibrous tissue stroma. The round window membrane encircled the electrode. Furthermore, in the inflamed middle ear there was no transgression of the round window membrane or electrode sheath.

Both the structure and characteristics of the mesothelial lining and fibrous tissue, however, require further elucidation. Any changes in it using different electrode materials and over longer time periods require further study. Furthermore, permeability studies of the implanted round window membrane in the presence of otitis media also need to be performed.

Although a study by Franz et al. (1984) has shown that the area between the electrode and round window membrane is permeable to the horseradish peroxidase molecule this does not mean that organisms will enter via this route. In fact in this study spontaneously occurring infections in three cats did not lead to inner ear involvement in cochleae which had been previously implanted. Furthermore, in this study by Franz et al. a Silastic ${ }^{\circledR}$ tube without encircling platinum bands was used. Silastic ${ }^{\circledR}$ leads to a mesothelial lining with poor adherence to the material, and a potential space between the two. It was therefore a 'worst case' situation. On the other hand, we have observed no mesothelium around platinum bands, and better tissue adherence. The bands could therefore act as a barrier to infection, and as they are also part of our human electrodes (Clark et al. 1979), they were used in the present study. This present study has helped confirm that following a cochlear implant with this banded electrode an induced otitis media does not lead to the spread of infection to the vital inner ear structures, when staphylococci are inoculated into the middle ear.

\section{Conclusions}

A combined technique of middle ear inoculation with staphylococci and Eustachian tube obstruction, was found to be a successful means of inducing an inflammatory response in the middle ear of the experimental animal. Furthermore, it induces a middle ear infection 
in a way that is similar to the pathogenesis of otitis media, and it does not disturb a previously implanted cochlear electrode. It therefore meets the requirements of a technique needed to investigate more thoroughly the effects of middle ear infection on the implanted cochlea.

In the study, the normal round window membrane was shown to resist the spread of infection from the middle ear to the cochlea. In the event of an intracochlear implant these protective features were retained.

\section{Acknowledgements}

We would like to acknowledge the financial support provided by the National Health and Medical Research Council of Australia and the Deafness Foundation of Victoria. We wish to thank Mr. David Bloom for carrying out the histology and photography and Mrs. H. Hodgens for typing the manuscript.

\section{References}

Clark, G. M., Patrick, J. F. and Bailey, Q. R. (1979) A cochlear implant round window electrode array. Journal of Laryngology and Otology, 93: 107-109.

Clark, G. M. and ShePHERD, R. K. (1984) Cochlear implant round window sealing procedures in the cat-an investigation of autograft and heterograft materials. Acta Otolaryngologica, Supplement, 410: 5-15.

Franz, B., Clark, G. M. and Bloom, D. M. (1984) Permeability of the implanted round window membrane in the cat-an investigation using horseradish peroxidase. Acta Otolaryngologica, Supplement, 410: 17-23.

Giebink, G. S., Berzins, I. K. and Quie, P. G. (1980) Animal models for studying pneumococcal otitis media and pneumococcal vaccine efficacy. Annals of Otology, Rhinology and Laryngology, 89 (Pt 2 Suppl. 68): 339343.

Goycoolea, M. V. (1983) Pathogenesis of otitis media. Ear Clinics International, 3: 1-2.

Goycoolea, M. V., Paparella, M. and Juhn, S. K. (1980) Oval and round window changes in otitis media: potential pathways between middle and inner ear. Laryngoscope, 90: 1387-1391.

Kawabata, I. and Paparella, M. (1971) Fine structure of the round window membrane. Annals of Otology, Rhinology and Laryngology, 80: 13-25.

KHAN, J. A. and Campbell, J. C. (1981) Studies on the production of middle ear effusion in the experimental animal. Journal of Laryngology and Otology, 95: 987-993.

Paparella, M., Goycoolea, M. and Meyerhoff, W. (1980) Inner ear pathology and otitis media. Annals of Otology, Rhinology and Laryngology, Supplement, 89: 249-253.

Shepherd, R. K., Webb, R. L., Clark, G. M., Pyman, B. C., Hirshorn, M. S., Murray, M. T. and Houghton. M. E. (1984) Implanted material tolerance studies for a multiple-channel cochlear prosthesis. Atta Otolaryngologica, Supplement, 411: 71-81.

Professor Graeme M. Clark,

Department of Otolaryngology.

University of Melbourne.

Parkville, Victoria 3052

Australia. 


\section{University Library}

\section{- M M I N E R VA A gateway to Melbourne's research publications}

Minerva Access is the Institutional Repository of The University of Melbourne

Author/s:

Brennan, Warwick J.;Clark, Graeme M.

Title:

An animal model of acute otitis media and the histopathological assessment of a cochlear implant in the cat

Date:

1985

Citation:

Brennan, W. J., \& Clark, G. M. (1985). An animal model of acute otitis media and the histopathological assessment of a cochlear implant in the cat. Journal of Laryngology and Otology, 99(9), 851-856.

Persistent Link:

http://hdl.handle.net/11343/27214 